\title{
Tumour-associated macrophages mediate the invasion and metastasis of bladder cancer cells through CXCL8
}

\author{
Hao Wu ${ }^{1,2,3}$, Xiangxiang Zhang ${ }^{1,2,3}$, Dali Han ${ }^{1,2,3}$, Jinlong Cao ${ }^{1,2,3}$, Junqiang Tian ${ }^{\text {Corresp. 1, 2, } 3}$ \\ 1 Department of Urology, Lanzhou University Second Hospital, Lanzhou, Gansu, P.R. China \\ 2 Urology Institute, Lanzhou University Second Hospital, Lanzhou, Gansu, P.R. China \\ ${ }^{3}$ Key Laboratory of Gansu Province for Urological Diseases, Lanzhou, Gansu, P.R. China \\ Corresponding Author: Junqiang Tian \\ Email address: ery_tianjq@Izu.edu.cn
}

Tumour-associated macrophages (TAMs) are associated with both the progression and poor prognosis of a variety of solid tumours. This study aimed to investigate and clarify the tumour-promoting role of CXCL8 secreted by TAMs in the urothelial carcinoma microenvironment of the bladder. Immunohistochemistry $(n=55)$ was used to detect Chemokine (C-X-C motif) ligand 8 (CXCL8), CD163 (a TAM marker), Matrixmetalloproteinase-9 (MMP-9), vascular endothelial growth factor (VEGF), and Ecadherin in cancerous and adjacent tissues of bladder cancer patients. TAMs-like PBM (peripheral blood mononuclear)-derived macrophages were developed using in vitro experiments. T24, 5637, and UM-UC-3 were treated with conditioned medium (CM) for the experimental intervention group, without $\mathrm{CM}$ for the blank control group, and with $\mathrm{CM}$ and an anti-CXCL8 neutralizing antibody for the experimental control group, respectively. The immunohistochemical study showed that the expression of CXCL8 was significantly upregulated as the number of infiltrating TAMs increased in the tumour tissues. A high expression of CXCL8 significantly correlated with an increase in the expression of MMP-9 and VEGF and a decrease in expression of E-cadherin in the microenvironment. This revealed that TAM-derived CXCL8 is highly associated with bladder cancer migration, invasion, and angiogenesis. The concentration of CXCL8 was significantly higher in CM collected from TAM-like PBM-derived macrophages than that from THP-1 cells. In subsequent in vitro experiments, we found that CM derived from TAM-like PBM-derived macrophages can also increase the migration rate, invasiveness, and pro-angiogenic properties of tumour cells. Additionally, the effect of CXCL8 was significantly diminished by the addition of an anti-CXCL8 neutralizing antibody to CM. The infiltration of TAMs in the tumour microenvironment leads to the elevation of CXCL8, which in turn promotes the secretion of MMP-9, VEGF, and E-cadherin by bladder cancer cells. This alters the migration, invasion, and pro-angiogenic capacity of bladder cancer cells and accelerates 
cancer progression. 
1 Tumour-associated macrophages mediate the invasion and metastasis of bladder cancer cells through

2 CXCL8

3 Hao Wu, Xiangxiang Zhang, Dali Han, Jinlong Cao, Junqiang Tian

4 Department of Urology of Lanzhou University Second Hospital, Urology Institute of Lanzhou University Second

5 Hospital, Key Laboratory of Gansu Province for Urological Diseases, Lanzhou, Gansu, P.R. China.

6 Corresponding Author: Junqiang Tian, Lanzhou, Gansu, PR China

7 E-mail: ery_tianjq@1zu.edu.cn

8

9

10

11

12

13

14

15

16

17

Peer] reviewing PDF | (2019:10:42475:1:1:NEW 25 Jan 2020) 


\section{Abstract}

of CXCL8 significantly correlated with an increase in the expression of MMP-9 and VEGF and a decrease in 
cells and accelerates cancer progression.

\section{INTRODUCTION} been found to be indeed involved in tumorigenesis. (Gordon et al. 2017). In the tumour microenvironment, macrophages with the M2 phenotype polarized by IL-4, IL-10, etc., are often referred to as "tumour-associated macrophages" (TAMs). TAMs are a major component in leukocyte infiltration in tumour tissues (Hao et al. 2012; Noy \& Pollard 2014). CD163 (a hemoglobin scavenger receptor) is a unique TAM marker (Almatroodi et al. 2016; Barros et al. 2013). After recruiting tumour-derived chemokines (mainly Chemokine (C-C motif) ligand 2 (CCL2) and colony-stimulating factor 1 (CSF-1) (Noy \& Pollard 2014)), TAMs are often confined to the perivascular region or the tumour-infiltrating margin. Upon arrival, they can secrete pro-migratory factors (e.g. epidermal growth factor, or EGF), degrade extracellular matrix proteolytic enzymes, accelerate tumour migration, and induce tumour invasion and metastasis (Huang \& Feng 2013; Pollard 2004; Quail \& Joyce 2013). It has been shown that in solid tumours, including bladder cancer, an increased number of $\mathrm{CD} 163^{+} \mathrm{TAM}$ infiltrates is positively correlated with a poor clinical outcome (Komohara et al. 2014; Lewis \& Pollard 2006; Wang et al. 2015).

Solid tumours and their surrounding areas are composed of inflammatory cells as well as various types of chemokines and cytokines (Stadler et al. 2015). CXCL8 is considered a prototypic chemokine belonging to the CXC angiogenesis, but also activates MMP to promote metastasis-related tissue remodeling (Azenshtein et al. 2005; Kim 
58 is significantly higher than that of healthy volunteers (Pignot et al. 2009; Sheryka et al. 2003), and the overexpression

59 of CXCL8 is positively correlated with significantly reduced survival of patients overall (Zhang et al. 2014). It has

60 been shown that TAMs in the solid tumour microenvironment can secrete CXCL8 in large amounts, directly

61 contributing to the progression of pancreatic ductal carcinoma, papillary thyroid cancer, colorectal cell carcinoma, and esophageal squamous cell carcinoma (Chen et al. 2018; Fang et al. 2014; Hosono et al. 2017; Lee et al. 2012). on bladder cancer cell production.

\section{2. MATERIALS AND METHODS}

(H\&E)-stained specimens obtained by initial cystectomy were reassessed independently by experienced uropathologists. 
was reached, the sections were incubated in the pressure cooker for $2 \mathrm{~min}$. Endogenous peroxidase activity was blocked using $1 \%$ hydrogen peroxide in methanol according to the Streptavidin-Peroxidase Detection Kit (ZSGB$\mathrm{BIO}$, China) after blocking in $1 \%$ bovine serum albumin (BSA) for $10 \mathrm{~min}$. Slides were incubated overnight at $4{ }^{\circ} \mathrm{C}$ with anti-human CXCL8 antibody (Abcam, CA, goat polyclonal, diluted 1/1000 in 1\% BSA), anti-CD163 antibody, anti-VEGF antibody, anti-MMP-9 antibody, and anti-E-cadherin antibody (Santa Cruz, CA, goat polyclonal, diluted $1 / 250$ in $1 \%$ BSA), respectively. Slides were then incubated with biotinylated anti-goat IgG secondary antibody for $10 \mathrm{~min}$ at room temperature. Sections were subsequently stained with 3, 3'-diaminobenzidine (DAB; Vector Laboratories), counterstained with hematoxylin (Solarbio, Peking, China), dehydrated, and coverslipped. The results were observed under a microscope (OLYMPUS BH-2 Microscopes, USA), and the positive staining rate was counted. The semi-quantitative methods used for immunohistochemistry results were as follows: CD163-positive round cells were counted in cancerous areas using at least three independent high-power microscopic fields (HPF; 400×, 0.0625 $\mu \mathrm{m}^{2}$ ). The mean number of CD163-positive cells was considered the number of tumour-infiltrating TAMs. To quantify the expression levels of CXCL8, MMP9, VEGF, and E-cadherin in bladder cancer cells, tumour cells were counted in at least three independent fields (HPF; $400 \times, 0.0625 \mu \mathrm{m}^{2}$ ), and that number was divided by the total cancer cells to calculate the percentage of positive cells $(1-100 \%)$. The evaluation was carried out blindly by two investigators, without knowledge of the patients' outcome or other clinic pathological characteristics.

\subsection{Cell lines and reagents}

Three bladder cancer cell lines, 5637, UM-UC-3, and T24, as well as acute monocytic leukemia cell line THP-1 and EA·hy926 (Cell Bank of the Chinese Academy of Sciences, Peking, China) were used in the present study. Cell lines were maintained in RPMI-1640 medium (Gibco, Thermo Fisher Scientific, USA) supplemented with 10\% fetal 
97

98

99

100

101

102

103

104

105

106

107

108

109

110

111

112

113

114

115

116 bovine serum (Pan Biotech, Germany), 100 units $/ \mathrm{mL}$ penicillin, and $100 \mu \mathrm{g} / \mathrm{mL}$ streptomycin in a standard humidified incubator at $37^{\circ} \mathrm{C}$ in $5 \% \mathrm{CO}_{2}$. Phorbol-12-myristate-13-acetate (PMA) was purchased from Multis Sciences (LIANKE) Biotech (Hangzhou, China). Recombinant protein IL-4 (PeproTech, USA) was also used in the described experiments.

\subsection{Generation of TAMs from THP-1}

To generate TAMs from monocytic THP-1, cells were seeded $1 \times 10^{6}$ cells in a dish and treated with PMA (200nM) for $24 \mathrm{~h}$ for differentiation into resting macrophages (M0 cells). For M2 polarization, cells were treated with IL-4 (20 $\mathrm{ng} / \mathrm{mL}$ ) for an additional $48 \mathrm{~h}$ (Genin et al. 2015; Mantovani et al. 2004). qRT-PCR analysis for CD163 was performed to confirm TAM generation.

2.5 Enzyme-linked immunosorbent assay (ELISA)

A human CXCL8 kit and MMP-9 kit were purchased from Neobioscience Technology Company, China, and ELISA was performed according to the manufacturer's instructions. Both standard and tissue samples were added to a 96-well plate with $0.1 \mathrm{~mL}$ of biotinylated antibody and incubated at $37^{\circ} \mathrm{C}$ for 60 minutes. After gentle washing, enzyme conjugate working solution was added to each well and the samples incubated for $30 \mathrm{~min}$. Chromogenic substrate (TMB) was then added and the samples incubated for $15 \mathrm{~min}$ in the dark. After stopping with stop solution, the optical density at $450 \mathrm{~nm}$ was quantified using a microplate reader (Bio Tek Instruments, USA) for further analysis. 2.6 Quantitative real-time polymerase chain reaction (qRT-PCR)

RNA was extracted from cells using TRIzol (Takara, Japan) as per the manufacturer's instructions. Conversion to cDNA was achieved using cDNA PrimeScript ${ }^{\mathrm{TM}}$ RT Master Mix (Perfect Real Time) (Takara, Japan) in a reverse transcription PCR instrument (Bio-Rad Laboratories, USA). Quantitative qRT-PCR was carried out using the CFX

Peer) reviewing PDF | (2019:10:42475:1:1:NEW 25 Jan 2020) 
117 Real-Time PCR System (Bio-Rad Laboratories, USA) in a $15-\mu 1$ reaction volume containing first-strand cDNA, TB

118 Green $^{\mathrm{TM}}$ Premix Ex Taq ${ }^{\mathrm{TM}}$ II (Tli RNaseH Pluse). Primer sets can be found in Table 1. Relative fold changes in mRNA

119 levels were calculated after normalization to GADPH using the comparative $\mathrm{Ct}$ method.

$120 \quad 2.7$ Western blot analysis

121 After the treated cells were collected and washed with PBS and RIPA buffer lysis, they were placed on ice for 30

122 minutes. The concentration of supernatant protein was determined using the BCA method (Solarbio, Peking, China)

123 after centrifugation at a speed of 14,000 r.p.m. for 15 minutes. The same amount of total protein was resolved using

124 SDS-PAGE and transferred to the PVDF membrane. Antibodies to the target protein were tested overnight at $4^{\circ} \mathrm{C}$.

125 The primary antibodies used in this study were anti-E-cadherin (dilution,1:100), anti-VEGF (dilution,1:200), and anti-

126 actin antibody (dilution, 1:1000; ZSGB-BIO, China) as an internal loading control. After incubation with IRDye ${ }^{\circledR}$

near-infrared fluorescence secondary antibody, the protein was detected using Odyssey Near-Infrared Fluorescence

Imaging Systems (LI-COR Biosciences, USA).

2.8 Cell invasion assay using conditioned media obtained from TAMs

134 according to the manufacturer's directions, and 24-well plates with 8 - $\mu \mathrm{m}$ pore membranes were lightly coated with

$1352 \mathrm{mg} / \mathrm{mL}$ Matrigel (Solarbio, Peking, China). T24, 5637, and UM-UC-3 cells were added to each insert at a density of

13630,000 cells/well in the CM described above. The lower chamber contained RPMI1640 medium with 10\% FBS as a 
141 Cells were seeded in six-well plates and incubated overnight. A vertical bar was drawn using a mark pen on the

142 back of the six-well plate, and a $1 \mathrm{~mL}$ pipette tip was used to transversely scratch the cell monolayer. After washing

143 the scraped cells from the wells with PBS, serum-free medium, CM, and CM with added anti-CXCL8 neutralizing

144 antibody were added to the wells. Tumour cell migration was observed at $0,6,12$ and $24 \mathrm{~h}$. Wound healing percentage

145 was calculated using Image J software.

1462.10 Vascular endothelial cell tube formation experiment

147 Matrigel $(8.7 \mathrm{mg} / \mathrm{mL})$ was placed in a refrigerator at $4^{\circ} \mathrm{C}$ to slowly melt overnight, and $50 \mu \mathrm{L}$ was spread in each

148 well of a 96 -well plate and placed in an incubator at $37^{\circ} \mathrm{C}$ for 1 hour. The EA-hy926 cells were prepared into a cell

149 suspension of $2 \times 10^{5}$ cells $/ \mathrm{mL}$, which was mixed well and added to a $96-$ well plate at a volume of $100 \mu \mathrm{L}$ per well. The

$150 \mathrm{CM}$ prepared in advance was then added at a volume of $50 \mu \mathrm{L}$ per well, incubated in an incubator at $37{ }^{\circ} \mathrm{C}$ and $5 \%$

$151 \mathrm{CO}_{2}$, and observed after 8 hours (Arnaoutova \& Kleinman 2010; Lee \& Kang 2018; Ma et al. 2017). We observed the

152 tube formation of endothelial cells and counted the number of vessels and visible nodes.

$153 \quad 2.11$ Statistical Analysis

154 All experimental results were expressed as mean \pm SD and analyzed for statistical significance by a two-tailed

155 Student's $t$-test. The interrelationship was examined using Spearman's analysis. Data were statistically analyzed and 
156 plotted using IBM SPSS version 23 (SPSS Inc., Chicago, IL, USA) and Prism software version 5 (San Diego, CA,

157 USA), respectively. A $p$-value $<0.05$ was considered statistically significant.

\section{3. RESULTS}

3.1 The association between the expression of CXCL8 in bladder cancer and clinicopathological features 
176 of IL-4 in vitro induce different amounts of CXCL8 secreted by TAM-like PBM-derived macrophages (Figure 2D).

177 The highest amount of CXCL8 was secreted when the concentration of IL-4 was 20 ng/mL (Figure 2E). After

178 analyzing the expression of CXCL8 and CD163 using qRT-PCR, we concluded that CXCL8 expression significantly

179 increased (Figure 2F) and TAM-like PBM-derived macrophages induced by IL-4 significantly expressed the specific

180 M2 macrophage marker CD163 (Figure 2G).

1813.3 CXCL8 derived from TAM-like PBM-derived macrophages promotes the invasion of bladder cancer

182 To examine the potential consequences of recruiting macrophages into bladder cancer tumour microenvironments,

183 we investigated the potential effect of TAM-derived CXCL8 on bladder cancer cell invasion. We tested invasion

184 ability after culturing bladder cancer cells 5637, T24, and UM-UC-3 with/without CM, using $8 \mu \mathrm{m}$ membrane inserts

185 as described in the Materials and Methods. The results show that CM increases bladder cancer cells' invasion capacity,

186 and this effect can be suppressed using an anti-CXCL8 neutralizing antibody (Figure 3A-J). RNA subsequently

187 extracted from bladder cancer cells after the CM treatment described above showed that the expression of MMP-9

188 had significantly increased in the treated cells (Figure 3K). This was also demonstrated by ELISA at the protein level

189 (Figure 3L), indicating that CXCL8 can promote bladder cancer invasion by stimulating bladder cancer cells to secrete

190 MMP-9.

1913.4 CXCL8 derived from TAM-like PBM-derived macrophages increases the migration of bladder cancer cells

192 We conducted a wound healing experiment to investigate the effect of CXCL8 on bladder cancer cell migration.

193 We planted 5637, T24, and UM-UC-3 bladder cancer cells on a six-well plate, attached them to the wall for 24 hours,

194 and then added CM (Figure 4A-R). We found that the cell migration increased significantly after the addition of CM. 
195 After neutralizing CXCL8 in CM with anti-CXCL8 neutralizing antibody, the accelerated migration rate was observed

196 to be significantly reduced. This suggests that CXCL8 can promote the migration of cancer cells (Figure 4S).

197 3.5 CXCL8 derived from TAM-like PBM-derived macrophages promotes the formation of blood vessels in tumours

198 Angiogenesis is associated with the benign-malignant transition of tumours, with resulting blood vessels providing

\section{4. DISCUSSION}


It has been well-established that the microenvironment of solid tumors is constituted of malignant cells and some non-malignant mesenchymal cells (Balkwill \& Mantovani 2001; Nilendu et al. 2018). Among these nonmalignant cells, macrophages play an important role in promoting tumour migration, invasion, and new blood vessel formation. TAMs in the tumour microenvironment can lead to pro-tumorigenic inflammation, which plays an important role in tumorigenesis (Mantovani \& Sica 2010), but TAMs can also express both chemokines and cytokines to promote an immunosuppressive tumour microenvironment (Guo et al. 2016). The infiltration of TAMs in primary tumours is associated with a poorer prognosis across almost all tumours (Mantovani et al. 2017), and when macrophage infiltration in the tumour microenvironment is blocked using a colony stimulating factor-1 (CSF-1) or colony stimulating factor-1 receptor (CSF-1R) inhibitor, the tumour burden in patients can be significantly reduced (Ries et al. 2014). Although it has been established that TAMs are associated with the progression of solid tumours and poor patient prognoses, the specific TAM mechanism in tumour cells has not been fully explained. This study found that the infiltration of TAMs in bladder cancer patients significantly increased

bladder cancer tissues. This demonstrates that a high expression of CXCL8 is strongly associated with the infiltration

of TAMs, and studies have shown similar findings in other cancers (Fang et al. 2014; Lin et al. 2019). 
235 CXCL8, vascular endothelial cells begin an angiopoiesis process characterized by the secretion of MMP which

236 breaks down the extracellular matrix ( $\mathrm{Li}$ et al. 2003). CXCL8 can significantly increase tumour vessel density

237 in prostate cancer, which has a very strong angiopoiesis effect (Araki et al. 2007; Inoue et al. 2000a; Masuya et

238 al. 2001). In the present study, we observed that the high expression of CXCL8 in bladder cancer tissues

239 positively correlated with the elevated expression of VEGF, suggesting that CM derived in vitro from TAM-like

240 PBM can promote the secretion of VEGF from bladder cancer cells and blood vessel formation. Therefore, the

241 synergistic effect of CXCL8 and VEGF can promote tumour angiogenesis (Masuya et al. 2001).

242 CXCL8-induced MMP-9 promotes extracellular matrix degradation (Inoue et al. 2000b), which not only

243 provides the necessary conditions for angiogenesis, but also plays an important role in cancer cell invasion and

244 metastasis. In the present study, a high expression of MMP-9 in cancer tissues was observed by

CM derived from TAM-like PBM-derived CXCL8 could contribute to MMP-9 expression in bladder cancer 
255 after extracting RNA and protein from bladder cancer cells treated with and without CM, we found that the

256

257

258

259

260

261

262

263

264

265

266

267

268

269

270

271

272

microenvironment on tumors.

273

(Figure 4T-V).

CONCLUSION expression of E-cadherin significantly decreased in bladder cancer cells treated with CM, an effect that could be

resisted using an anti-CXCL8 neutralizing antibody (Among bladder cancer cells, T24 has the highest degree of

malignancy, which may lead to low or even no expression of E-cadherin in T24). This demonstrates that CXCL8

derived from TAMs can increase bladder cancer cell migration by decreasing the expression of E-cadherin

This study found that infiltrating TAMs can modify the bladder tumor microenvironment by promoting tumor

progression with CXCL8. A contrasting study has shown that CXCL8 secreted by tumor cells activates

proangiogenic and anti-apoptotic pathways by promoting the expression of VEGFA and bcl-2 at the invasion

front, which confirms the key role of CXCL8 in tumor progression (Kumar et al. 2018). A similar autocrine

mechanism of this CXC chemokine has also been reported (Bandapalli et al. 2011; Matsuo et al. 2009). By

observing immunohistochemical section staining, we found that bladder cancer cells could also secrete CXCL8,

meaning that there is complex intercellular communication in the tumor microenvironment involving autocrine

and paracrine chemokines. Due to the complexity of the tumor microenvironment and its components, tumor

invasion, migration, and angiogenesis may be determined by more than one factor or pathway. Therefore, a

macroscopic view of the tumor microenvironment would be effective. This study focused on the role of CXCL8

secreted by TAMs in promoting bladder cancer, but further work is needed to determine the full effect of

Peer) reviewing PDF | (2019:10:42475:1:1:NEW 25 Jan 2020) 
In summary, TAM-derived CXCL8 can promote the expression of MMP-9, VEGF, and E-cadherin in bladder

cancer cells, causing changes in bladder cancer cell migration, invasion, and pro-angiogenic ability, and leading

to the progression of bladder cancer. The inhibition of CXCL8-signaling derived from TAMs may be a promising

therapeutic approach in the treatment of bladder cancer.

\section{Acknowledgements}

\section{None}

\section{Reference}

Almatroodi SA, McDonald CF, Darby IA, and Pouniotis DS. 2016. Characterization of M1/M2 TumourAssociated Macrophages (TAMs) and Th1/Th2 Cytokine Profiles in Patients with NSCLC. Cancer Microenviron 9:1-11. 10.1007/s12307-015-0174-x

Araki S, Omori Y, Lyn D, Singh RK, Meinbach DM, Sandman Y, Lokeshwar VB, and Lokeshwar BL. 2007. Interleukin- 8 is a molecular determinant of androgen independence and progression in prostate cancer. Cancer Res 67:6854-6862. 10.1158/0008-5472.can-07-1162

Arnaoutova I, and Kleinman HK. 2010. In vitro angiogenesis: endothelial cell tube formation on gelled basement membrane extract. Nat Protoc 5:628-635. 10.1038/nprot.2010.6

Azenshtein E, Meshel T, Shina S, Barak N, Keydar I, and Ben-Baruch A. 2005. The angiogenic factors CXCL8 and VEGF in breast cancer: regulation by an array of pro-malignancy factors. Cancer Lett 217:73-86. 10.1016/j.canlet.2004.05.024

Balkwill F, and Mantovani A. 2001. Inflammation and cancer: back to Virchow? Lancet 357:539-545. 10.1016/s0140-6736(00)04046-0

Bandapalli OR, Hariri N, Macher-Goppinger S, Kahlert C, Schirmacher P, and Brand K. 2011. Increased apoptosis of host cells and tumor cells in the invasion front of colorectal liver metastases. Anticancer Res 31:1215-1224.

Barros MH, Hauck F, Dreyer JH, Kempkes B, and Niedobitek G. 2013. Macrophage polarisation: an immunohistochemical approach for identifying M1 and M2 macrophages. PLoS One 8:e80908. 10.1371/journal.pone.0080908

Cao Y. 2005. Tumor angiogenesis and therapy. Biomed Pharmacother 59 Suppl 2:S340-343.

Chen SJ, Lian GD, Li JJ, Zhang QB, Zeng LJ, Yang KG, Huang CM, Li YQ, Chen YT, and Huang KH. 2018. Tumor-driven like macrophages induced by conditioned media from pancreatic ductal adenocarcinoma promote tumor metastasis via secreting IL-8. Cancer Med 7:5679-5690. 10.1002/cam4.1824

Cheng XS, Li YF, Tan J, Sun B, Xiao YC, Fang XB, Zhang XF, Li Q, Dong JH, Li M, Qian HH, Yin ZF, 
306

307

308

309

310

311

312

313

314

315

316

317

318

319

320

321

322

323

324

325

326

327

328

329

330

331

332

333

334

335

336

337

338

339

340

341

342

343

344

345

346

and Yang ZB. 2014. CCL20 and CXCL8 synergize to promote progression and poor survival outcome in patients with colorectal cancer by collaborative induction of the epithelialmesenchymal transition. Cancer Lett 348:77-87. 10.1016/j.canlet.2014.03.008

Fang W, Ye L, Shen L, Cai J, Huang F, Wei Q, Fei X, Chen X, Guan H, Wang W, Li X, and Ning G. 2014. Tumor-associated macrophages promote the metastatic potential of thyroid papillary cancer by releasing CXCL8. Carcinogenesis 35:1780-1787. 10.1093/carcin/bgu060

Genin M, Clement F, Fattaccioli A, Raes M, and Michiels C. 2015. M1 and M2 macrophages derived from THP-1 cells differentially modulate the response of cancer cells to etoposide. BMC Cancer 15:577. 10.1186/s12885-015-1546-9

Gordon SR, Maute RL, Dulken BW, Hutter G, George BM, McCracken MN, Gupta R, Tsai JM, Sinha R, Corey D, Ring AM, Connolly AJ, and Weissman IL. 2017. PD-1 expression by tumour-associated macrophages inhibits phagocytosis and tumour immunity. Nature 545:495-499. 10.1038/nature22396

Guo Q, Jin Z, Yuan Y, Liu R, Xu T, Wei H, Xu X, He S, Chen S, Shi Z, Hou W, and Hua B. 2016. New Mechanisms of Tumor-Associated Macrophages on Promoting Tumor Progression: Recent Research Advances and Potential Targets for Tumor Immunotherapy. J Immunol Res 2016:9720912. 10.1155/2016/9720912

Ha H, Debnath B, and Neamati N. 2017. Role of the CXCL8-CXCR1/2 Axis in Cancer and Inflammatory Diseases. Theranostics 7:1543-1588. 10.7150/thno.15625

Hao NB, Lu MH, Fan YH, Cao YL, Zhang ZR, and Yang SM. 2012. Macrophages in tumor microenvironments and the progression of tumors. Clin Dev Immunol 2012:948098. 10.1155/2012/948098

Hosono M, Koma YI, Takase N, Urakawa N, Higashino N, Suemune K, Kodaira H, Nishio M, Shigeoka M, Kakeji Y, and Yokozaki H. 2017. CXCL8 derived from tumor-associated macrophages and esophageal squamous cell carcinomas contributes to tumor progression by promoting migration and invasion of cancer cells. Oncotarget 8:106071-106088. 10.18632/oncotarget.22526

Huang YC, and Feng ZP. 2013. The good and bad of microglia/macrophages: new hope in stroke therapeutics. Acta Pharmacol Sin 34:6-7. 10.1038/aps.2012.178

Inoue K, Slaton JW, Eve BY, Kim SJ, Perrotte P, Balbay MD, Yano S, Bar-Eli M, Radinsky R, Pettaway CA, and Dinney CP. 2000a. Interleukin 8 expression regulates tumorigenicity and metastases in androgen-independent prostate cancer. Clin Cancer Res 6:2104-2119.

Inoue K, Slaton JW, Kim SJ, Perrotte P, Eve BY, Bar-Eli M, Radinsky R, and Dinney CP. $2000 \mathrm{~b}$. Interleukin 8 expression regulates tumorigenicity and metastasis in human bladder cancer. Cancer Res 60:2290-2299.

Kim SJ, Uehara H, Karashima T, McCarty M, Shih N, and Fidler IJ. 2001. Expression of interleukin-8 correlates with angiogenesis, tumorigenicity, and metastasis of human prostate cancer cells implanted orthotopically in nude mice. Neoplasia 3:33-42. 10.1038/sj.neo.7900124

Komohara Y, Jinushi M, and Takeya M. 2014. Clinical significance of macrophage heterogeneity in human malignant tumors. Cancer Sci 105:1-8. 10.1111/cas.12314

Kumar A, Cherukumilli M, Mahmoudpour SH, Brand K, and Bandapalli OR. 2018. ShRNA-mediated knock-down of CXCL8 inhibits tumor growth in colorectal liver metastasis. Biochem Biophys Res 
Commun 500:731-737. 10.1016/j.bbrc.2018.04.144

Lee H, and Kang KT. 2018. Advanced tube formation assay using human endothelial colony forming cells for in vitro evaluation of angiogenesis. Korean $J$ Physiol Pharmacol 22:705-712. 10.4196/kjpp.2018.22.6.705

Lee YS, Choi I, Ning Y, Kim NY, Khatchadourian V, Yang D, Chung HK, Choi D, LaBonte MJ, Ladner RD, Nagulapalli Venkata KC, Rosenberg DO, Petasis NA, Lenz HJ, and Hong YK. 2012. Interleukin- 8 and its receptor CXCR2 in the tumour microenvironment promote colon cancer growth, progression and metastasis. Br J Cancer 106:1833-1841. 10.1038/bjc.2012.177

Lewis CE, and Pollard JW. 2006. Distinct role of macrophages in different tumor microenvironments. Cancer Res 66:605-612. 10.1158/0008-5472.can-05-4005

Li A, Dubey S, Varney ML, Dave BJ, and Singh RK. 2003. IL-8 directly enhanced endothelial cell survival, proliferation, and matrix metalloproteinases production and regulated angiogenesis. J Immunol 170:3369-3376. 10.4049/jimmunol.170.6.3369

Lin C, He H, Liu H, Li R, Chen Y, Qi Y, Jiang Q, Chen L, Zhang P, Zhang H, Li H, Zhang W, Sun Y, and $\mathrm{Xu}$ J. 2019. Tumour-associated macrophages-derived CXCL8 determines immune evasion through autonomous PD-L1 expression in gastric cancer. Gut. 10.1136/gutjnl-2018-316324

Lin EY, and Pollard JW. 2007. Tumor-associated macrophages press the angiogenic switch in breast cancer. Cancer Res 67:5064-5066. 10.1158/0008-5472.can-07-0912

Liu Q, Li A, Tian Y, Wu JD, Liu Y, Li T, Chen Y, Han X, and Wu K. 2016. The CXCL8-CXCR1/2 pathways in cancer. Cytokine Growth Factor Rev 31:61-71. 10.1016/j.cytogfr.2016.08.002

Ma L, Li G, Cao G, Zhu Y, Du MR, Zhao Y, Wang H, Liu Y, Yang Y, Li YX, Li DJ, Yang H, and Wang YL. 2017. dNK cells facilitate the interaction between trophoblastic and endothelial cells via VEGF-C and HGF. Immunol Cell Biol 95:695-704. 10.1038/icb.2017.45

Mantovani A, Marchesi F, Malesci A, Laghi L, and Allavena P. 2017. Tumour-associated macrophages as treatment targets in oncology. Nat Rev Clin Oncol 14:399-416. 10.1038/nrclinonc.2016.217

Mantovani A, and Sica A. 2010. Macrophages, innate immunity and cancer: balance, tolerance, and diversity. Curr Opin Immunol 22:231-237. 10.1016/j.coi.2010.01.009

Mantovani A, Sica A, Sozzani S, Allavena P, Vecchi A, and Locati M. 2004. The chemokine system in diverse forms of macrophage activation and polarization. Trends Immunol 25:677-686. 10.1016/j.it.2004.09.015

Masuya D, Huang C, Liu D, Kameyama K, Hayashi E, Yamauchi A, Kobayashi S, Haba R, and Yokomise H. 2001. The intratumoral expression of vascular endothelial growth factor and interleukin-8 associated with angiogenesis in nonsmall cell lung carcinoma patients. Cancer 92:2628-2638.

Matsuo Y, Raimondo M, Woodward TA, Wallace MB, Gill KR, Tong Z, Burdick MD, Yang Z, Strieter RM, Hoffman RM, and Guha S. 2009. CXC-chemokine/CXCR2 biological axis promotes angiogenesis in vitro and in vivo in pancreatic cancer. Int $J$ Cancer 125:1027-1037. $10.1002 /$ ijc. 24383

Mukaida N. 2003. Pathophysiological roles of interleukin-8/CXCL8 in pulmonary diseases. Am J Physiol Lung Cell Mol Physiol 284:L566-577. 10.1152/ajplung.00233.2002

Nilendu P, Sarode SC, Jahagirdar D, Tandon I, Patil S, Sarode GS, Pal JK, and Sharma NK. 2018. Mutual concessions and compromises between stromal cells and cancer cells: driving tumor development 
and drug resistance. Cell Oncol (Dordr) 41:353-367. 10.1007/s13402-018-0388-2

Noy R, and Pollard JW. 2014. Tumor-associated macrophages: from mechanisms to therapy. Immunity 41:49-61. 10.1016/j.immuni.2014.06.010

Pignot G, Bieche I, Vacher S, Guet C, Vieillefond A, Debre B, Lidereau R, and Amsellem-Ouazana D. 2009. Large-scale real-time reverse transcription-PCR approach of angiogenic pathways in human transitional cell carcinoma of the bladder: identification of VEGFA as a major independent prognostic marker. Eur Urol 56:678-688. 10.1016/j.eururo.2008.05.027

Pollard JW. 2004. Tumour-educated macrophages promote tumour progression and metastasis. Nat Rev Cancer 4:71-78. 10.1038/nrc1256

Quail DF, and Joyce JA. 2013. Microenvironmental regulation of tumor progression and metastasis. Nat Med 19:1423-1437. 10.1038/nm.3394

Ries CH, Cannarile MA, Hoves S, Benz J, Wartha K, Runza V, Rey-Giraud F, Pradel LP, Feuerhake F, Klaman I, Jones T, Jucknischke U, Scheiblich S, Kaluza K, Gorr IH, Walz A, Abiraj K, Cassier PA, Sica A, Gomez-Roca C, de Visser KE, Italiano A, Le Tourneau C, Delord JP, Levitsky H, Blay JY, and Ruttinger D. 2014. Targeting tumor-associated macrophages with anti-CSF-1R antibody reveals a strategy for cancer therapy. Cancer Cell 25:846-859. 10.1016/j.ccr.2014.05.016

Sheryka E, Wheeler MA, Hausladen DA, and Weiss RM. 2003. Urinary interleukin-8 levels are elevated in subjects with transitional cell carcinoma. Urology 62:162-166.

Stadler M, Walter S, Walzl A, Kramer N, Unger C, Scherzer M, Unterleuthner D, Hengstschlager M, Krupitza G, and Dolznig H. 2015. Increased complexity in carcinomas: Analyzing and modeling the interaction of human cancer cells with their microenvironment. Semin Cancer Biol 35:107-124. 10.1016/j.semcancer.2015.08.007

Wang B, Liu H, Dong X, Wu S, Zeng H, Liu Z, Wan D, Dong W, He W, Chen X, Zheng L, Huang J, and Lin T. 2015. High CD204+ tumor-infiltrating macrophage density predicts a poor prognosis in patients with urothelial cell carcinoma of the bladder. Oncotarget 6:20204-20214. 10.18632/oncotarget.3887

Waugh DJ, and Wilson C. 2008. The interleukin-8 pathway in cancer. Clin Cancer Res 14:6735-6741. 10.1158/1078-0432.ccr-07-4843

Zhang G, Gomes-Giacoia E, Dai Y, Lawton A, Miyake M, Furuya H, Goodison S, and Rosser CJ. 2014. Validation and clinicopathologic associations of a urine-based bladder cancer biomarker signature. Diagn Pathol 9:200. 10.1186/s13000-014-0200-1 
Table $\mathbf{1}$ (on next page)

qRT-PCR primers (human) 


\section{Tables 1}

2 qRT-PCR primers (human)

\begin{tabular}{|l|l|l|}
\hline CXCL8 & Forward & CAAGCTGGCCGTGGCTCT \\
\hline CD163 & Forward & TGGGGTGGAAAGGTTTGGAGT \\
\hline & Reverse & GGCCTCCTTTTCCATTCCAGAAA \\
\hline MMP-9 & Forward & GTACTCGACCTGTACCAGCG \\
\hline VEGF & Feverse & TTCAGGGCGAGGACCATAGA \\
\hline & Reverse & ATTTGTTGTGCTGTAGGAAGCT \\
\hline E-cadherin & Forward & ATGGCTGAAGGTGACAGAGC \\
\hline & Reverse & CACCTTCCATGACAGACCCC \\
\hline GADPH & Forward & CAGGA GGCAT TGCTG ATGAT \\
\hline & Reverse & GAAGG CTGGG GCTCA TTT \\
\hline
\end{tabular}


Table 2 (on next page)

Clinicopathologic characteristics of 55 cases with bladder cancer 
Table 2.

Clinicopathologic characteristics of 55 cases with bladder cancer

\begin{tabular}{|c|c|c|c|c|}
\hline \multirow[t]{2}{*}{ Variables } & \multirow{2}{*}{ Cases } & \multicolumn{2}{|c|}{$\begin{array}{l}\text { CXCL8 expression in tumor } \\
\text { Number of cases (percentage) }\end{array}$} & \multirow[t]{2}{*}{$P$} \\
\hline & & Low & High & \\
\hline Total & 55 & 18 & 37 & \\
\hline Age & & & & 0.78 \\
\hline$<65$ & 29 & $9(30 \%)$ & $20(70 \%)$ & \\
\hline$\geq 65$ & 26 & $9(35 \%)$ & $17(65 \%)$ & \\
\hline Sex & & & & 0.31 \\
\hline Male & 44 & $13(30 \%)$ & $31(70 \%)$ & \\
\hline Female & 11 & $5(45 \%)$ & $6(55 \%)$ & \\
\hline Tumour size & & & & 0.41 \\
\hline less than $3 \mathrm{~cm}$ & 9 & $4(44 \%)$ & $5(56 \%)$ & \\
\hline $3 \mathrm{~cm}$ and more & 46 & $14(30 \%)$ & $32(70 \%)$ & \\
\hline Tumour stage & & & & $0.01^{*}$ \\
\hline$<\mathrm{T} 2$ & 13 & $9(69 \%)$ & $4(31 \%)$ & \\
\hline$\geq \mathrm{T} 2$ & 42 & $9(21 \%)$ & $33(79 \%)$ & \\
\hline TumoUr grade & & & & $0.02^{*}$ \\
\hline Low grade & 6 & $4(65 \%)$ & $2(35 \%)$ & \\
\hline High grade & 49 & $11(22 \%)$ & $36(78 \%)$ & \\
\hline Lymph node metastasis & & & & 0.13 \\
\hline Negative & 23 & $10(44 \%)$ & $13(56 \%)$ & \\
\hline Positive & 33 & $8(25 \%)$ & $25(75 \%)$ & \\
\hline Vascular invasion & & & & 0.73 \\
\hline No & 38 & $13(42 \%)$ & $25(66 \%)$ & \\
\hline Yes & 17 & $5(29 \%)$ & $12(71 \%)$ & \\
\hline Distant metastasis & & & & 0.93 \\
\hline No & 31 & $10(33 \%)$ & $21(67 \%)$ & \\
\hline Yes & 24 & $8(31 \%)$ & $16(69 \%)$ & \\
\hline
\end{tabular}

$1 \quad P$ value was analyzed by a chi-square test; $*$ indicates $P<0.05$ with statistical significance. 


\section{Figure 1}

Recruitment of tumor-associatedmacrophages and CXCL8 expression in human bladder cancer, and the correlation between the expression of CXCL8 and the expression of MMP-9, VEGF, and E-cadherin.

(A-J) Representative expression status of CXCL8, CD163, MMP-9, VEGF and E-cadherin in human bladder cancer and para-cancertissues. All images were captured at $100 x$ and $400 x$ magnification. (K) Expression of CXCL8 in bladder cancer and para-cancertissues. In the boxand-whisker plot, significance was assessed by the paired student's $t$ test. $* * * * p<0.0001$. (LO) The interrelationship between the percentage of CXCL8 positive cancer cells and (L) the number of infiltrated TAM, (M) percentage of MMP-9 positive cells, (N) percentage of VEGF positive cells, and ( 0 ) percentage of E-cadherin positive cells using Spearman's correlation. Spearman $r$ was found to be $0.41,0.47,0.57,-0.42$, respectively. (95\% confidence interval, 0.16-0.61, 0.24-0.66, 0.36-0.73, -0.61- -0.17, respectively). 


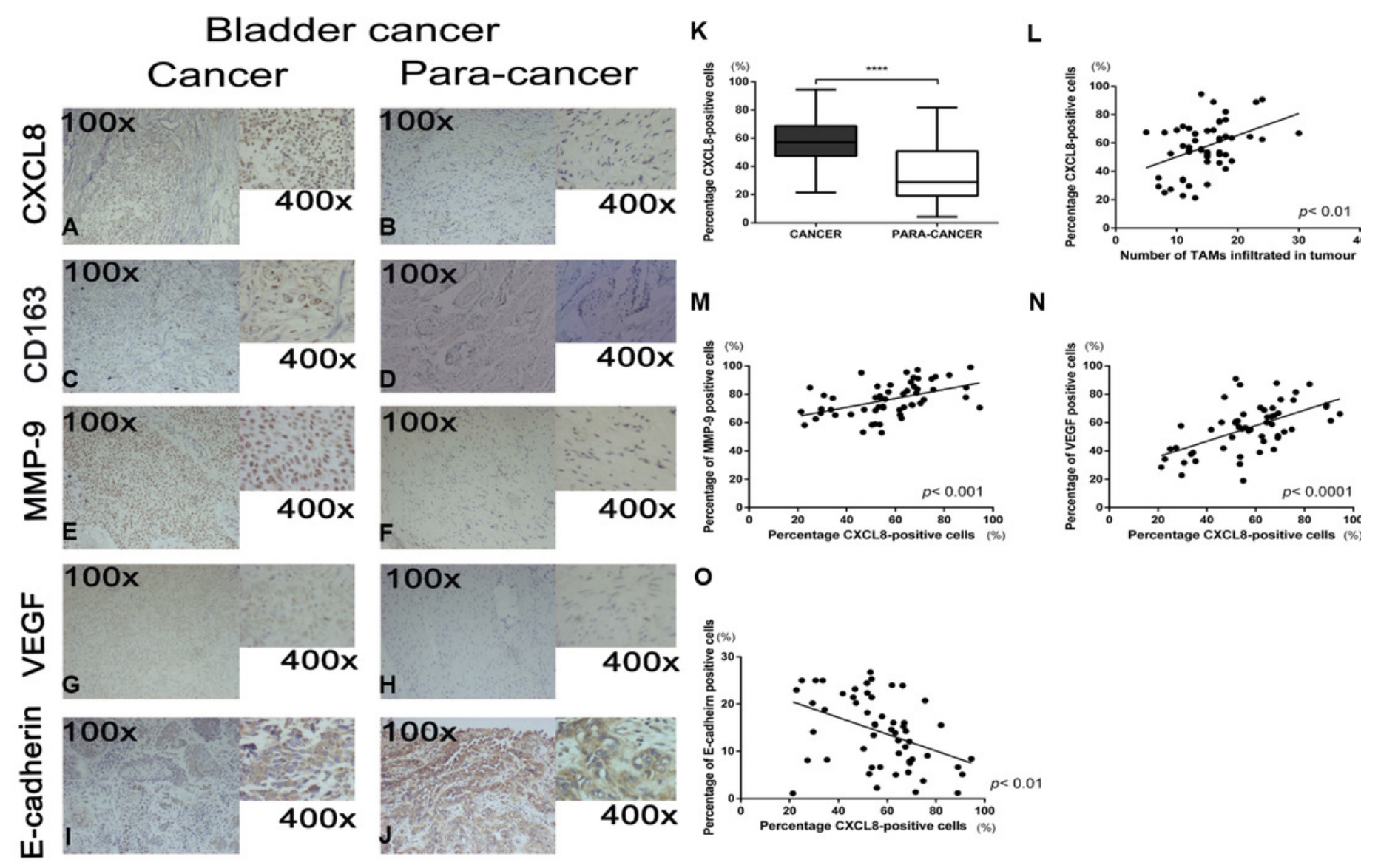




\section{Figure 2}

IL-4-induced TAM-like PBM-derived macrophages can secrete CXCL8.

(A-C) Morphological changes of THP-1. PMA (200nM, 24h) and IL-4 (20ng/mL, 48h)

sequentially induce the formation of TAMs-like PBM-derived macrophages. Prior to treatment, THP-1 cells were round, floating, and did not attach to the bottom surface of the flask; after treatment with PMA and IL-4, they adhered to the bottom surface with abundant cytoplasmic processes. (D) After M0 induction using different concentrations of IL-4, the concentration of CXCL8 in serum-free medium was measured over time by ELISA. ${ }^{* *} p<0.01,{ }^{* * *} p<0.001$. (E) When the concentration of IL-4 was $20 \mathrm{ng} / \mathrm{mL}$, the amount of CXCL8 secreted by TAMslike PBM-derived macrophages was the greatest. (F) CXCL8 mRNA expression level was determined by qRT-PCR. (G) The expression level of CD163 in TAM-like PBM-derived macrophages. Expression levels are expressed as the mean \pm SD. $* p<0.05, * * p<0.01$, *** $p<0.001$. 


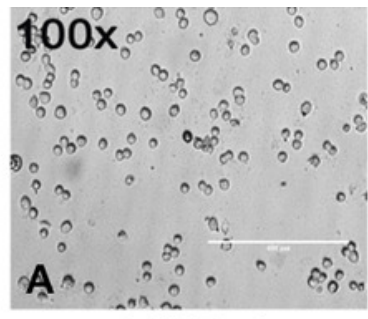

THP-1

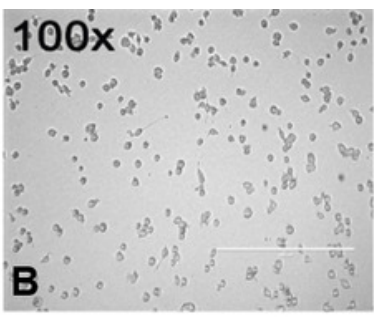

200nM PMA

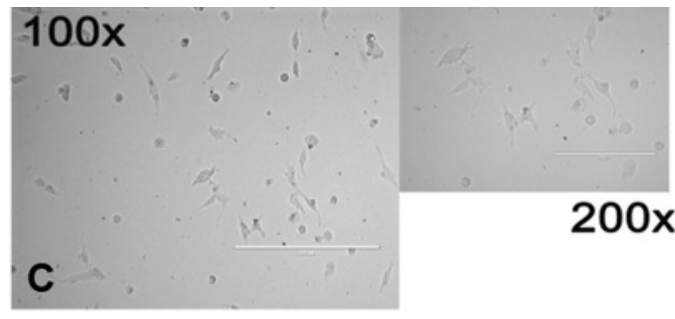

20ng/mL IL-4

E

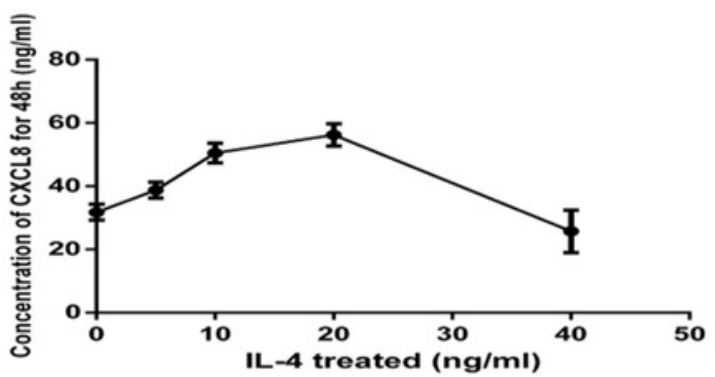

G

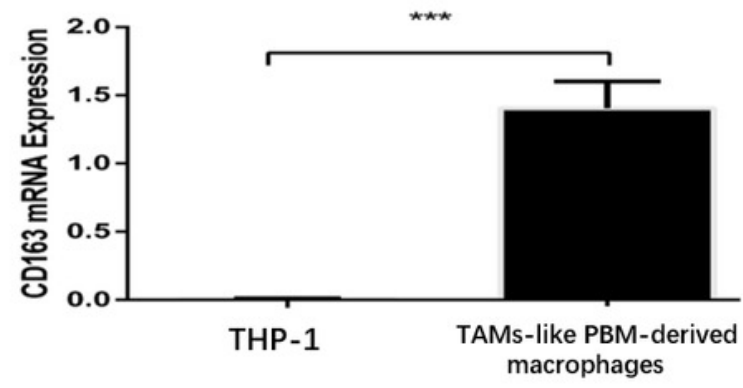




\section{Figure 3}

TAM-like PBM-derived macrophages increase the invasiveness of bladder cancer cells through CXCL8.

(A-I) For Transwell invasion assays, 5637, T24, and UM-UC-3 cells were seeded at $5.0 \times 10^{5}$ cells/well on a Matrigel-coated Transwell chamber in serum-free RPMI-1640. Serum-free RPMI-1640, CM, and CM with anti-CXCL8 neutralizing antibody were added to the Transwell chamber. The cell insert was placed on a 24-well plate containing RPMI-1640 with 10\% FBS for $48 \mathrm{~h}$. The invasive cells on the membrane were stained and counted. (J) The number of bladder cancer invasive cells on the membrane (1, Control; 2, CM; 3, CM with anti-CXCL8 neutralizing antibody). The results were expressed as the mean \pm SD. ${ }^{*} p<0.05$. (K-L) The expression level of MMP-9 in 5637, T24, UM-UC-3 was determined by (K) qRT-PCR and (L) ELISA (1, Control; 2, CM; 3, CM with anti-CXCL8 neutralizing antibody). Expression levels are expressed as the mean $\pm \mathrm{SD}$. $* p<0.05$. 

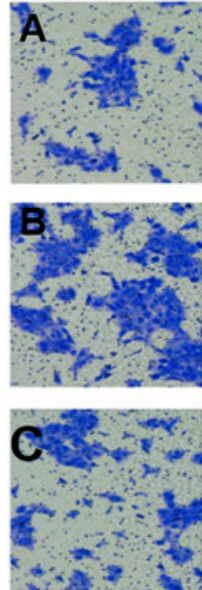

5637
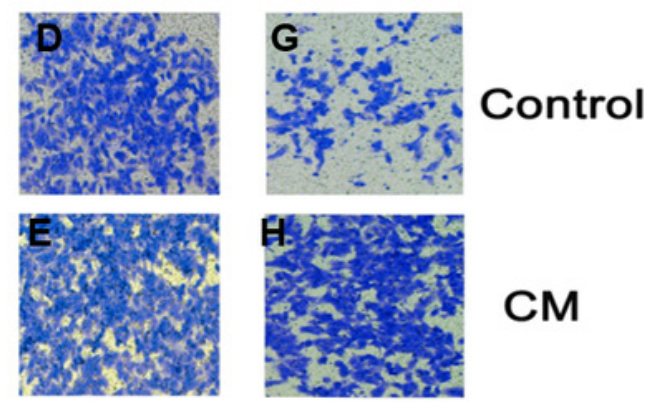

CM
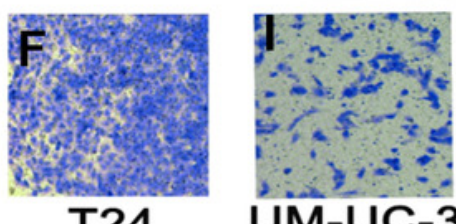
$\mathrm{CM}$ and anti- CXCL8 neutralizing antibody
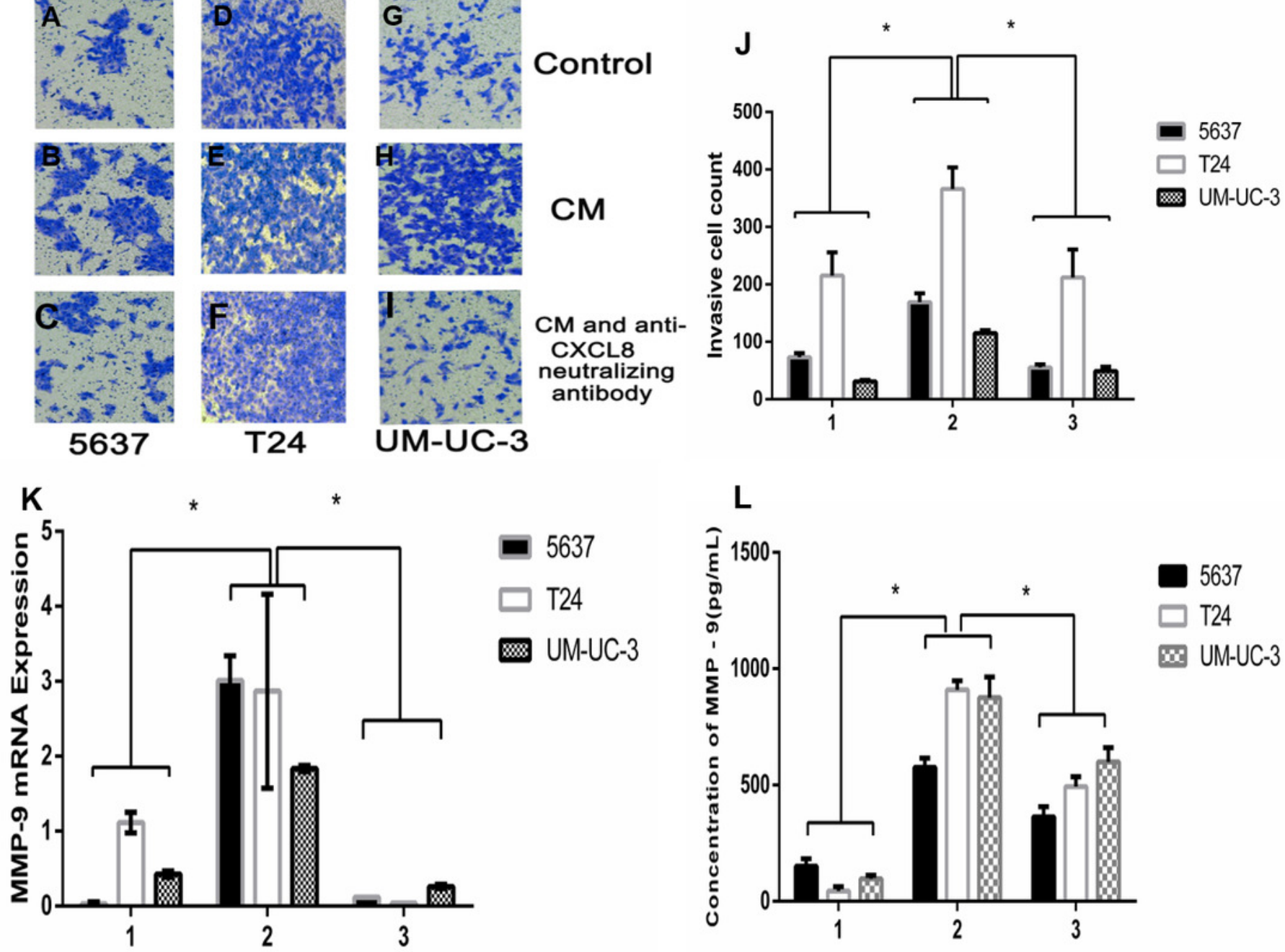


\section{Figure 4}

AM-like PBM-derived macrophagesincrease the migration of bladder cancer cells through CXCL8.

$\mathbf{T}(\mathrm{A}-\mathrm{R})$ Three kinds of bladder cancer cell lines (5637, T24, and UM-UC-3) were treated with serum-free RPMI-1640, CM, and CM with anti-CXCL8 neutralizing antibody, respectively. Cell migration was observed at $0,6,12,18$, and 24 hours. Pictures taken at 0 and 24 hours. (S) Percentage of wound healing. Formula: wound healing percentage for a certain time (initial area minus area at a certain time point) to the initial area (1, Control; $2, \mathrm{CM} ; 3, \mathrm{CM}$ with antiCXCL8 neutralizing antibody). The results were expressed as the mean \pm SD. $* p<0.05, * * p$ $<0.01$. (T) The expression level of E-cadherin in 5637, T24, UM-UC-3 was determined by qRT-PCR (1, Control; 2, CM; 3, CM with anti-CXCL8 neutralizing antibody). Expression levels are expressed as the mean \pm SD. ${ }^{* * *} p<0.001$. (U-V) 5637, T24, UM-UC-3 cells in serumfree conditions were treated with control, CM and CM with anti-CXCL8 neutralizing antibody. Western blotting was conducted with total protein extracted from bladder cancer cell lines using specific antibodies against E-cadherin and $\beta$-actin. The results are mean \pm SD. ${ }^{*} p<$ $0.05,{ }^{* *} p<0.01$. $(1,2,3$ were 5637 treated with control, CM and CM with anti-CXCL8 neutralizing antibody; 4,5,6 were T24 treated with control, CM and CM with anti-CXCL8 neutralizing antibody; 7,8,9 were UM-UC-3 treated with control, CM and CM with anti-CXCL8 neutralizing antibody.) 

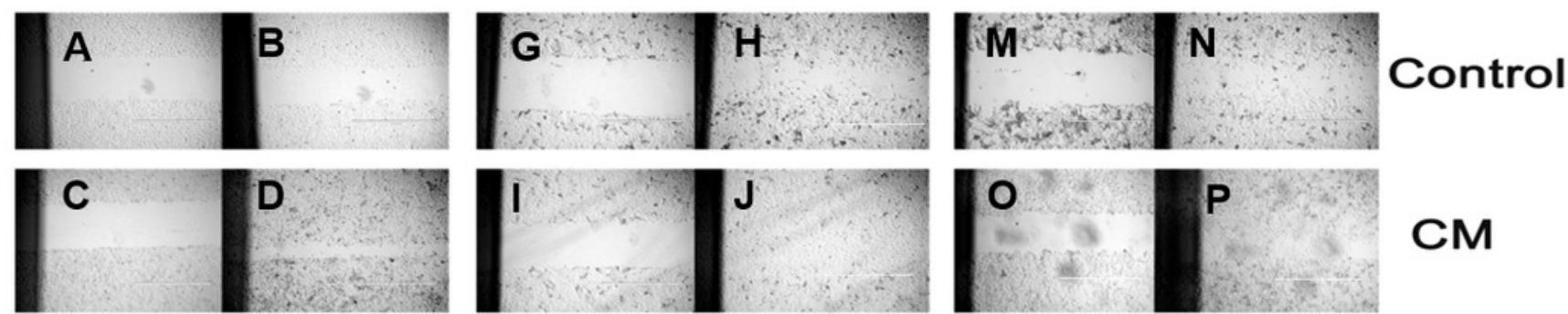

CM
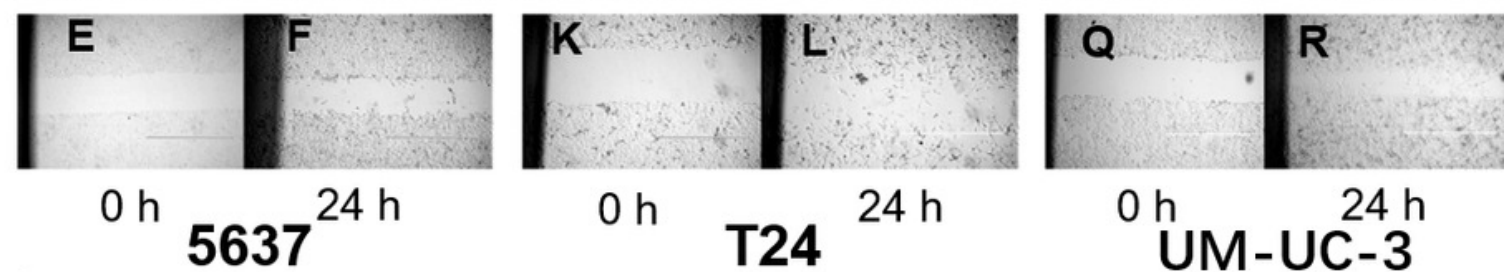

CM and anti-

CXCL8 neutralizing antibody

S

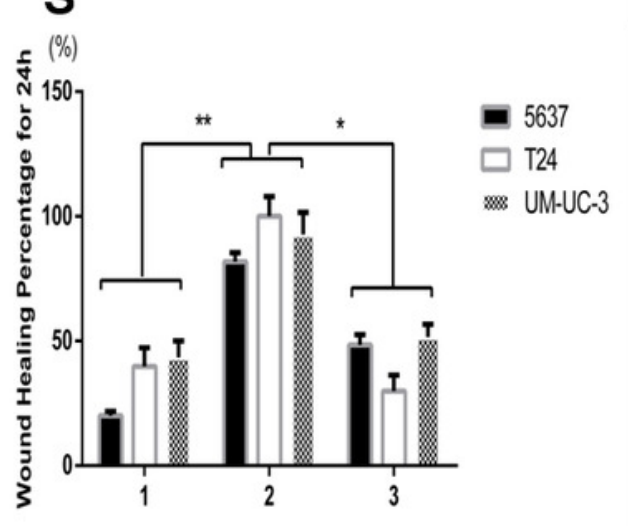

\section{UMM-UC-3}

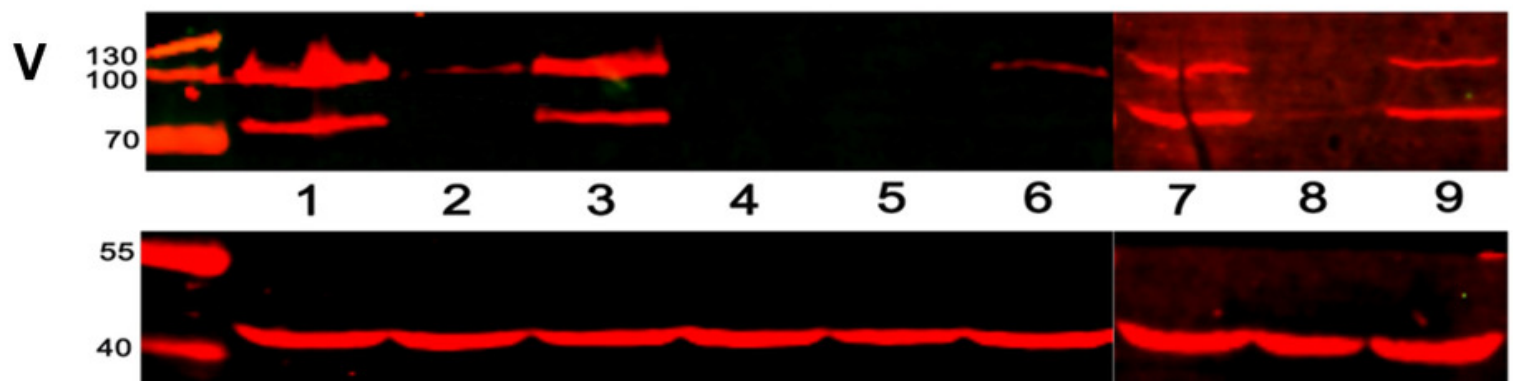

E-cadherin

$\beta$-actin 


\section{Figure 5}

CXCL8-derived TAM-like PBM-derived macrophagespromote the pro-angiogenic ability of bladder cancer cells.

(A-C) For endothelial cell tube formation experiments, EA.hy926 cells were added to a Matrigel-coated 96-well. CM-C, CM-T24, and CM-T24-NA were added to wells of a 96-well plate and the tube formation of endothelial cells was observed and photographed. (D) The number of vessels and nodes formed by EA.hy 926 cells was counted and plotted on the graph. The results were expressed as the mean $\pm \mathrm{SD}$. $* p<0.05$, $* * p<0.01$. (E) The expression level of VEGF in TAM-like PBM-derived macrophages was determined by qRT-PCR (1, Control; 2, CM; 3, CM with anti-CXCL8 neutralizing antibody). Expression levels are expressed as the mean $\pm \mathrm{SD}$. $* p<0.05, * * p<0.01$. (F-G) T24 cells were treated with serum-free medium, CM, and CM with anti-CXCL8 neutralizing antibody. The protein level of VEGF in the bladder cancer cell lines was confirmed by western blotting. Anti-VEGF and $\beta$ actin antibodies were used. The results are mean \pm SD. ${ }^{*} p<0.05,{ }^{* *} p<0.01 .(1,2,3$ were T24 treated with control, CM and CM with anti-CXCL8 neutralizing antibody.) 

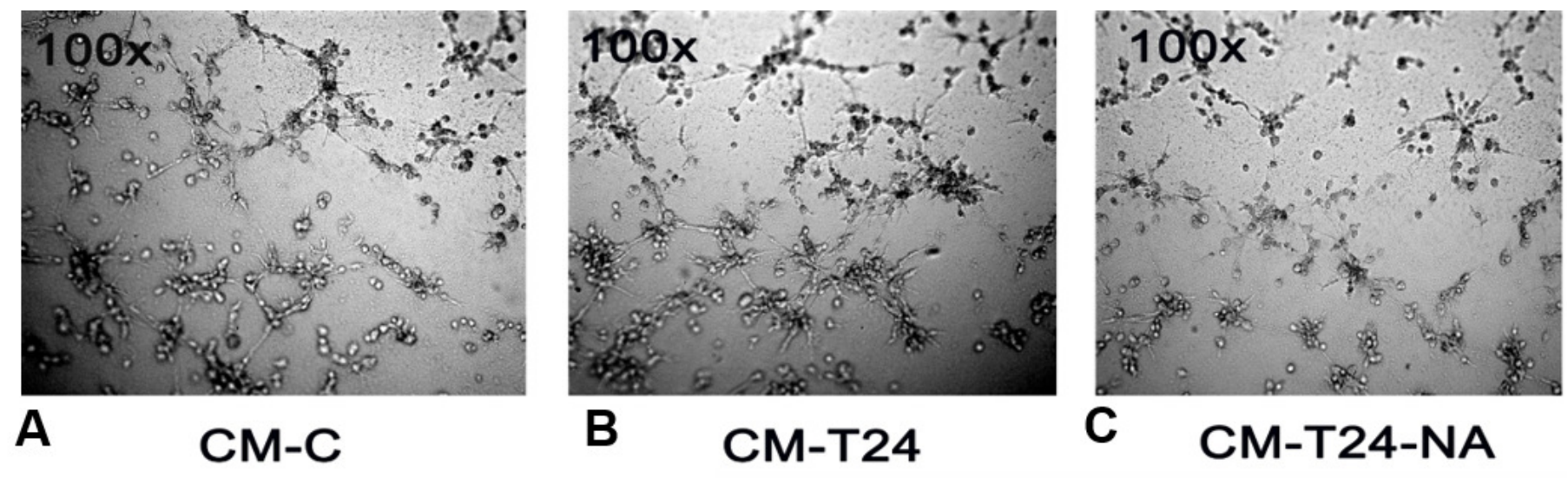

B

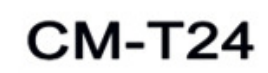

C CM-T24-NA

D

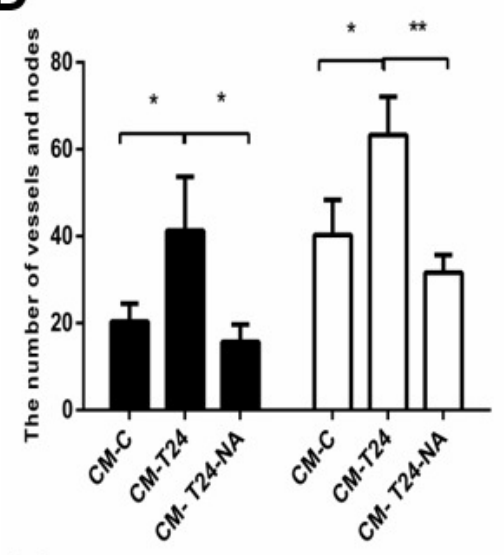

F

35

25

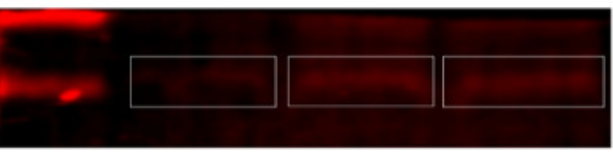

12

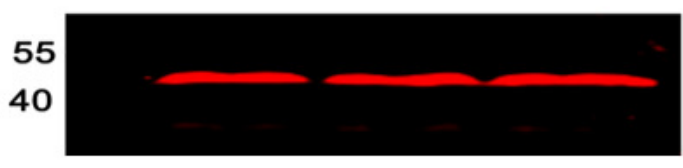

E

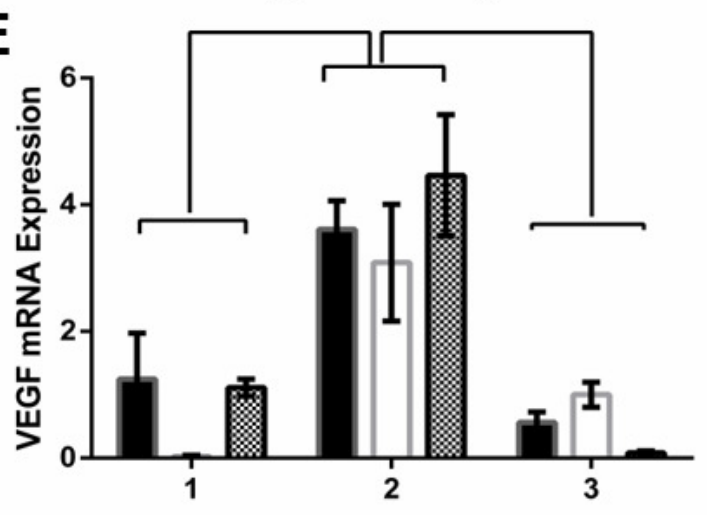

G

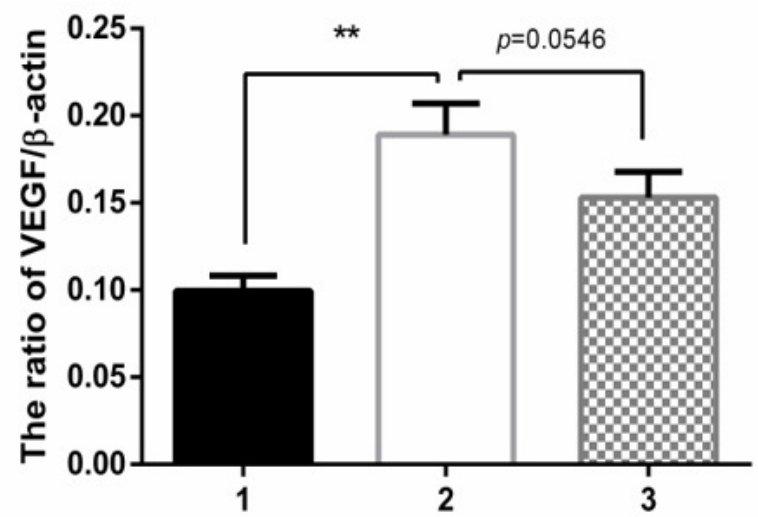

